\title{
Widespread Thalamic Terminations of Fibers Arising in the Superficial Medullary Dorsal Horn of Monkeys and Their Relation to Calbindin Immunoreactivity
}

\author{
Alessandro Graziano and Edward G. Jones \\ Center for Neuroscience, University of California Davis, Davis, California 95616
}

\begin{abstract}
The relay of pain fibers from the spinal and medullary dorsal horn in the thalamus has become a controversial issue. This study analyzed the relationship of fibers arising in lamina I to nuclei in and around the caudal pole of the ventral posterior nuclear complex and especially to a zone of calbindin-dense immunoreactivity (VMpo) identified by some authors as the sole thalamic relay for these fibers. We show that the densest zone of calbindin immunoreactivity is part of a more extensive, calbindin-immunoreactive region that lies well within the medial tip of the ventral posterior medial nucleus (VPM), as delineated by other staining methods, and prove that the use of different anti-calbindin antibodies cannot account for differences in interpretations of the organization of the posterior thalamic region. By combining immunocytochemical staining with anterograde tracing from injections involving lamina I, we demonstrate widespread fiber terminations that are not restricted to the calbindin-rich medial tip of VPM and show that the lamina I arising fibers are not themselves calbindin immunoreactive. This study disproves the existence of VMpo as an independent thalamic pain nucleus or as a specific relay in the ascending pain system.
\end{abstract}

Key words: calbindin; VMpo; spinothalamic; pain; thalamus; trigeminal

\section{Introduction}

The role of the thalamus in transmission of sensory messages relating to pain has become controversial. In the traditional view, spinothalamic (STT) and spinal trigemino-thalamic (sVTT) tract fibers terminate in widespread patches throughout the primate ventral posterior lateral (VPL), ventral posterior medial (VPM), and other posterior and intralaminar nuclei (Getz, 1952; Mehler et al., 1960; Price et al., 1976; Craig and Burton, 1981; Honda et al., 1983; Kniffki and Mizumura, 1983; Jones, 1985; Apkarian and Hodge, 1989a,b,c; Hodge and Apkarian, 1990; Rausell and Jones, 1991a,b; Rausell et al., 1992; Ralston and Ralston, 1994; Willis and Westlund, 1997). Cells with specific responses to noxious mechanical and thermal stimuli and those that project to the thalamus via the STT are located in the marginal zone and neck of the dorsal horn (laminas I and IV-VI), and substantial numbers of lamina I cells reportedly project to VPM and VPL (Willis et al., 2001). Neurons with pain- and temperature-specific stimulusresponse properties are described in most nuclei in which STT fibers terminate, including VPM and VPL (Applebaum et al., 1979; Casey and Morrow, 1983; Honda et al., 1983; Kenshalo and

\footnotetext{
Received Sept. 6, 2003; revised 0ct. 20, 2003; accepted 0ct. 27, 2003.

This work was supported by National Institutes of Health-United States Public Health Service Grants NS 21377 and NS 39094 and by the W. M. Keck Foundation Program in Neuroscience Imaging. We thank Phong L. Nguyen for technical help and Drs. T. M. Woods and F. Liang for assistance with the surgical procedure.

Correspondence should be addressed to Dr. Edward G. Jones, Center for Neuroscience, University of California Davis, Davis, CA 95616. E-mail: ejones@ucdavis.edu.

D0I:10.1523/JNEUROSCI.4122-03.2004

Copyright $\odot 2004$ Society for Neuroscience $\quad$ 0270-6474/04/240248-09\$15.00/0
}

Isensee, 1983; Chung et al., 1986; Chandler et al., 1992; Apkarian and Shi, 1994; Ralston and Ralston, 1994; Ohara and Lenz, 2003).

In a radical revision, Craig et al. (1994) and Blomqvist et al. (2000) proposed that thalamic terminations of axons arising from lamina I cells related to the whole body are restricted to a small focal area outside the confines of VPM and VPL and are strongly immunoreactive for the calcium-binding protein calbindin; they termed this region of highest calbindin expression VMpo. According to these authors, VMpo represents the thalamic nucleus specific for pain and temperature transmission in monkeys and humans, receives all inputs arising from lamina I, and relays this input to cingulate and insular cortex. Calbindin immunostaining was held to selectively delineate the thalamic pain relay and to specifically label the STT and sVTT.

This viewpoint stands in sharp contrast to descriptions of more widespread distributions of thalamic cells with pain- and temperature-specific responses cited above and to other descriptions of the cytoarchitecture and chemoarchitecture of the posterior thalamus in monkeys and humans (Jones and Hendry, 1989; Rausell and Jones, 1991a,b; Rausell et al., 1992; Morel et al., 1997; Jones et al., 2001). These showed that calbindin immunoreactivity is not confined to a single focus but extends throughout a number of nuclei of the posterior thalamus, all of which have been implicated in pain transmission. Craig et al. (1994) and Blomqvist et al. (2000) attributed the more restricted and selective character of their immunostaining for calbindin in comparison with other studies to the use of different antibodies.

The present study re-evaluates the neurochemical organization of the posterior thalamus and the relationship of sVTT fibers 
arising in lamina I to the region of highest calbindin expression called VMpo, the extent to which calbindin is expressed in these fibers, and the specificity of the anti-calbindin antibodies that have been held to be the source of differing interpretations of the chemical architecture of the posterior thalamus.

\section{Materials and Methods}

Surgery. This study was conducted on Macaca mulatta monkeys in accordance with the United States Public Health Service Policy on Humane Care and Use of Laboratory Animals and the National Institutes of Health's Guide for the Care and Use of Laboratory Animals.

The animals that underwent surgery $(n=3)$ were anesthetized with ketamine $(15 \mathrm{mg} / \mathrm{kg}$, i.m.) followed by continuous intravenous infusion of sodium pentobarbital ( $5 \mathrm{mg} / \mathrm{kg} / \mathrm{hr}$ ). Glass micropipettes (internal diameter, $10-20 \mu \mathrm{m})$ were filled with $10 \%$ fluorescein dextran $(10,000$ MW; Molecular Probes, Eugene, OR) dissolved in $0.9 \%$ saline. Silver wires were inserted into the internal solution to enable recording of multiunit responses to peripheral stimulation and to permit iontophoretic delivery of the tracer. The head was flexed, the lower medulla was exposed via the foramen magnum, and the micropipette was advanced into the left spinal trigeminal nucleus guided by recording multiunit responses to tactile stimulation of the face. Tracer was injected at a site within $100 \mu \mathrm{m}$ of the recording of the first cellular response. The tracer was ejected iontophoretically using $8 \mu \mathrm{A}$ cathodal current and a $50 \%$ duty cycle for $30 \mathrm{~min}$. After survival times of $20-22 \mathrm{~d}$, the animals were deeply anesthetized with Nembutal and perfused through the ascending aorta with $500 \mathrm{ml}$ of normal saline, followed by $4 \%$ paraformaldehyde in $0.1 \mathrm{~m}$ phosphate buffer, $\mathrm{pH}$ 7.4.

Histology. The brains were blocked, and the blocks were postfixed overnight, infiltrated with $30 \%$ sucrose in $0.1 \mathrm{~m}$ phosphate buffer for cryoprotection, and subsequently frozen in dry ice. Blocks of the brainstem and contralateral thalamus were sectioned serially at $30 \mu \mathrm{m}$ in the frontal Horsley-Clarke plane on a sliding microtome. All sections were collected in cold $0.1 \mathrm{~m}$ phosphate buffer. Every fourth (spinal cord and brainstem) and fifth (thalamus) section was mounted on glass slides and coverslipped with Vectashield (Vector Laboratories, Burlingame, CA) without additional processing for fluorescence detection. The remaining series of sections was processed alternatively for Nissl staining, cytochrome oxidase (CO) histochemistry (thalamus), or stained immunocytochemically for D-28k calbindin or parvalbumin.

Immunohistochemistry. The sections were preincubated for $1 \mathrm{hr}$ in blocking solution ( $0.1 \mathrm{~m}$ phosphate buffer, $0.25 \%$ Triton X-100, and $3 \%$ normal serum from the species in which the secondary antibodies were produced). After preincubation, the sections were incubated overnight in the same solution containing one of the following primary antibodies: polyclonal rabbit anti-calbindin (dilution, 1: 2000; a gift from Dr. P. C. Emson, Brabraham Institute, Cambridge, UK), monoclonal mouse anticalbindin (dilution, 1:3000; Sigma, St. Louis, MO), and monoclonal sheep anti-parvalbumin (dilution, 1:3000; Sigma). For double anticalbindin immunostaining, sections were incubated in phosphate buffer containing $0.25 \%$ Triton X-100, 3\% Chemiblocker (Chemicon, Temecula, CA), and both primary anti-calbindin antibodies in the above dilutions. After rinsing in phosphate buffer, the sections were transferred to the appropriate secondary IgG solutions and conjugated to one of the following: tetramethyl rhodamine isothiocyanate or fluorescein isothiocyanate (FITC) (Molecular Probes), Rhodamine Red-X (RRX; Jackson ImmunoResearch, West Grove, PA), Cy5 (Molecular Probes), or biotin (Vector Laboratories). For testing the relative staining capacities of the two anti-calbindin antibodies, RRX-, FITC-, and Cy5-conjugated secondary antibodies were used in different combinations with the two primary antibodies to control for possible affinity biases. The sections incubated with the fluorescent secondary antibodies were rinsed in phosphate buffer, mounted on glass slides, and coverslipped with Vectastain (Vector Laboratories). The sections incubated with the biotinylated secondary antibodies were rinsed in phosphate buffer, incubated in avidin-peroxidase complex (ABC; Vector Laboratories), rinsed in phosphate buffer, incubated in phosphate buffer containing $0.02 \% 3,3^{\prime}$ diaminobenzidine $4 \mathrm{HCl}$ (Sigma), and $0.03 \%$ hydrogen peroxide. After rinsing, the sections were mounted on glass slides, dried, dehydrated in increasing concentrations of alcohol, cleared in xylene, and coverslipped with a mixture of distyrene, tricresyl phosphate, and xylene mounting medium (BDH, Poole, England)

Data analysis. To determine the size and localization of the injections, projection drawings of the sections containing the injected dye were obtained by means of an epifluorescence microscope (Leitz, Rockleigh, $\mathrm{NJ}$ ), the stage of which was equipped with optical encoders interfaced to a personal computer using the Minnesota Datametrics (St. Paul, MN) MD2 plotting system. Similar outline drawings were obtained for the corresponding Nissl-stained sections. The plots of matching fluorescence and Nissl sections were imported into Adobe Photoshop 6 (Adobe Systems, San Jose, CA) and superimposed by means of Adobe Illustrator 9 (Adobe Systems) using section features and blood vessels as alignment landmarks. Additional photographic documentation of the tracer injections and their localization in the caudal spinal trigeminal nucleus (cSpV) was obtained by a dual-channel laser confocal scanning microscope (Fluoview-Olympus, Tokyo, Japan). Images of the thalamic sections were obtained either by confocal microscopy (see above), conventional photographic reversal film (Elite Chrome 400; Kodak, Rochester, NY) or by a Quantix CCD camera (PhotoMetrics, Tucson, AZ) interfaced to a personal computer and operated by SimplePCI software (Compix, Cranberry Township, PA). Image deconvolution was performed by using a module of the same software. Both latter image acquisition devices were attached to a Nikon (Tokyo, Japan) Eclipse 1000 microscope.

To identify and reconstruct the nuclear profile of the posterior thalamus, digital images of matching thalamic sections stained for Nissl or CO or immunostained for calbindin or parvalbumin were imported into Adobe Illustrator 9 and superimposed using section features and blood vessels as alignment landmarks. The thalamic nuclei were identified and their boundaries drawn on the basis of the staining patterns and in comparison with the atlases of Olszewski (1952) and Jones et al. (2001) using the revised terminology of Jones (1985) (available at http://neuroscience. ucdavis.edu/Jones/ThalamusImages/Monkey/). Subsequently, the plottings of the fluorescent fibers were imported into Adobe Illustrator 9, superimposed on the drawings of the thalamic nuclei and aligned as described above.

The analysis of calbindin expression in the ascending sVTT fibers and in the thalamic areas where they terminated was obtained by dualchannel laser confocal microscopy as above. The overall area of termination of labeled fibers in relation to the VP and adjacent nuclei was mapped by a collage of digitally captured images from multiple microscopic fields to reconstruct the whole area of fiber terminations. Matched images were obtained from adjacent sections histochemically stained for $\mathrm{CO}$; the two merged, and drawings of the outlines of the nuclei were superimposed.

Western blotting. The posterior thalamus from an unfixed rhesus monkey brain was dissected and homogenized on ice in lysis buffer $(150 \mathrm{~mm}$ $\mathrm{NaCl}, 20$ mm Tris-Cl, 2 mm EDTA, 1\% Triton X-100, 0.05\% SDS) with complete protease inhibitor mixture (Roche, Indianapolis, IN). The protein content was determined by the method of Bradford (1976), and equal amounts $(20 \mu \mathrm{g})$ were separated by $10 \%$ SDS-PAGE (Laemmli, 1970) using a Mini-PROTEAN 3 cell system (Bio-Rad, Hercules, CA). After electrophoresis, protein was transferred to polyvinylidene difluoride membranes (Amersham Biosciences, Piscataway, NJ) by an electrophoresis transfer system (Idea Scientific Company, Minneapolis, MN), washed briefly with Tris-buffered saline, $\mathrm{pH} 7.4$, containing $0.1 \%$ Tween 20 (TTBS), and blocked with 5\% skimmed milk in TTBS for $1 \mathrm{hr}$ at room temperature. Subsequently, the membranes were incubated overnight in TTBS with one of the two anti-calbindin antibodies at $4^{\circ} \mathrm{C}$. After washing in TTBS, the membranes were incubated in TTBS containing horseradish peroxidase-conjugated secondary antibodies (Sigma) for $1 \mathrm{hr}$ at room temperature, washed, and reacted with the chemiluminescence ECL system (Amersham Biosciences). Chemiluminescence was detected using a Molecular Dynamics Storm 860 phosphoimager and film autoradiography (Amersham Biosciences). 


\section{Results \\ Calcium-binding proteins in the thalamus}

Figure 1 illustrates the nuclear organization of the thalamus in the vicinity of the posterior pole of the ventral posterior complex, as delineated by Nissl and CO staining and by calbindin and parvalbumin immunostaining. The medial part of VPM is dominated by dense calbindin immunostaining. Of special note is the dense focus of calbindinimmunoreactive fibers and cells located close to the medial tip of the VPM nucleus (asterisk) but well within the boundaries of VPM as delineated by Nissl staining, by CO histochemical staining and by parvalbumin immunostaining. The borders of VPM are outlined by a thin fiber lamina visible in Nissl-, CO-, parvalbumin-, and calbindinstained preparations (Fig. 1). Enclosed within the lamina are the larger Nissl-stained cells that form the rod domain of the nucleus (Rausell and Jones, 1991a) and smaller cells of the matrix domain of VPM that include the small-celled zone of VPM and the basal ventral medial nucleus ( $\mathrm{VMb})$. The aggregated cells of the rods stain intensely for $\mathrm{CO}$ and for parvalbumin, and the rods at the medial tip of VPM also costain for calbindin, forming the densest focus of calbindin immunoreactivity in the posterior thalamus (Fig. 1, asterisk). This appears to be the region identified by others as VMpo. The matrix domain of VPM, including the VMb nucleus, stains selectively for calbindin. For series of sections stained in the same manner and extending through the rostrocaudal extent of VP and adjacent regions, see Jones et al. (2001) (also see http://neuroscience.ucdavis. edu/Jones/ThalamusImages/Monkey/).

\section{Localization of injections}

The injection of tracer in the superficial $\mathrm{cSpV}$ involved lamina I in all cases (Figs. 2A, 3), extending rostrocaudally for $1-1.5 \mathrm{~mm}$ (Fig. 2). To ensure staining of as many lamina I-arising fibers as possible, the injections were large enough to encroach on lamina II and sometimes lamina III as well. Figure 3, $A$ and $B$, shows a Nissl-stained section from the lower medulla and the staining of many calbindin-immunoreactive cells in laminas I and II of an adjacent section. Two tracks left by the recording-injecting electrodes are visible, ending in the superficial substantia gelatinosa; in the immunostained section, the discontinuity caused by the injection in the calbindin-rich superficial laminas of the $\mathrm{cSpV}$ indicates the involvement of laminas I and II. The extent of the injection of tracer and its relation to the laminas are shown in the two-channel confocal micrographs in Figure 3C-E. Laminas I-II and superficial lamina III are clearly identified by the dense calbindin immunostaining of cell bodies and neuropil (Fig. 3C,E, red). The deposit of tracer (Fig. 3D,E, green) is concentrated in lamina I and superficial lamina II, with less spread into lower lamina II and upper lamina III. Few cells of laminas II and III project to the thalamus (Willis et al., 2001); thus, the majority of ascending fibers labeled by these kinds of injections have their origins from cells located in lamina I. Figure $2 B$ gives a view of the course through the brainstem of sVTT fibers labeled by these injections, on the basis of the location of labeled fibers at selected cross-sectional levels in animal RM77.

\section{Nuclei in the thalamus and $\mathrm{cSpV}$ terminations}

Figure 4, from one of the brains, is representative of the results obtained in all three. In the contralateral thalamus, the terminations of labeled fibers arising from cells in the superficial dorsal horn are not confined to a single focal region or a single nucleus but are widely distributed across several nuclei in the posterior region. A dense cluster of terminations is concentrated toward the medial tip of VPM in all cases but well within the borders of the nucleus as delimited by the outlining fiber lamina. Many fibers approach this region through the caudal pole of the VPL nucleus. In Figure 5, plottings of the dense principal clusters of terminations were superimposed on images of adjacent COstained sections. These dense clusters of sVTT fiber terminations in VPM primarily occupy the parts of the nucleus between the dense CO-stained rods and corresponding to the matrix domain of VPM (Rausell and Jones, 1991a,b). Other substantial clusters of sVTT terminations are found in the posterior nucleus $(\mathrm{Po})$ and in caudal VPL; several scattered terminations of sVTT are also observed in the centre médian nucleus $(\mathrm{CM})$, ventral posterior inferior nucleus (VPI), VMb, and anterior pulvinar nucleus (Pla) (Fig. 4). 


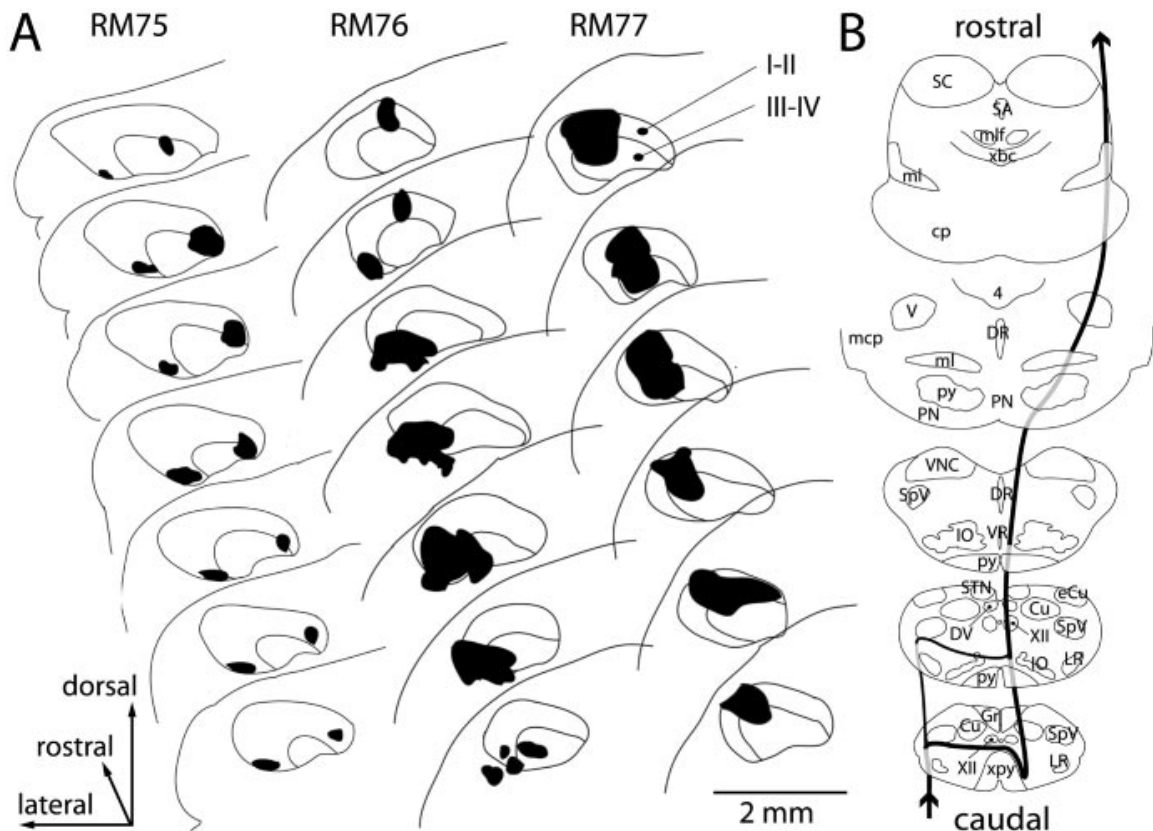

Figure 2. A, Serial plottings of the $\mathrm{CSpV}$ showing the extent and localization of the injection of tracer in the three animals (RM75, RM76, RM77). The tracer injections primarily involved the superficial-most laminas I and II in all three cases, with minor spread into lamina III. The rostrocaudal extent of the injections ranged between $1 \mathrm{~mm}$ (RM77) and $1.5 \mathrm{~mm}$ (RM76). B, Drawings of cross sections through the brainstem at selected medullary, pontine, and midbrain levels, showing the location of labeled ascending fibers (in RM77). 4, Fourth ventricle; cp, cerebral peduncle; Cu, cuneate nucleus; DR, dorsal raphe nucleus; DV, dorsal motor nucleus of the vagus; eCu, external cuneate nucleus; mlf, medial longitudinal fasciculus; Gr, gracile nucleus; I0, inferior olivary complex; LR, lateral reticular nucleus; mcp, middle cerebellar peduncle; ml, medial lemniscus; PN, pontine nuclei; py, pyramidal tract; SA, sylvian aqueduct; SC, superior colliculus; SpV, spinal trigeminal nucleus; STN, solitary tract nucleus; V, principal trigeminal nucleus; VNC, vestibular nuclear complex; VR, ventral raphe nucleus; xbc, decussation of the brachium conjunctivum; XII, hypoglossal nucleus; xpy, pyramidal decussation.

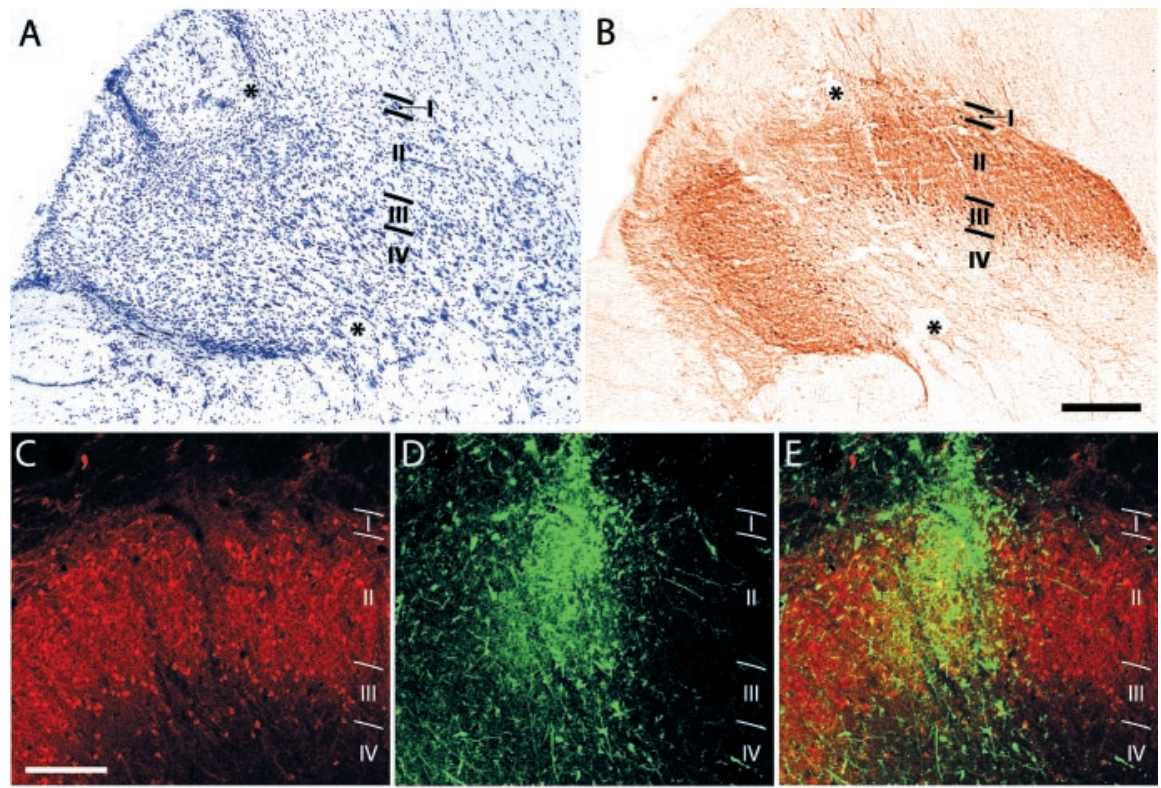

Figure 3. Localization of an injection in frontal sections of the $c S p V$ nucleus. $A$, Nissl-stained section showing two tracks left by the injecting-recording micropipette reaching the marginal zone (lamina I) and the superficial part of lamina II. $B$, Section adjacent to $A$, immunostained for calbindin. The discontinuity in the calbindin-rich superficial laminas of the $\mathrm{SpV}$ is caused by the injection of tracer and indicates the involvement of laminas I and II, primarily in its external portion. Asterisks in $A$ and $B$ mark the same blood vessel. $C-E$, Dual-channel laser confocal micrographs showing the site of injection of the fluorescent tracer $(D, E$, green) in relation to the calbindin immunostaining $(C-E$, red). Laminas I and II and the superficial part of lamina III are clearly identified by the dense calbindin immunolabeling of cell bodies and neuropil. The merged image in $E$ shows the deposit of tracer concentrated in lamina I and superficial lamina II, with less involvement of inner lamina II and minor involvement of lamina III. Scale bars: $A, B$, $300 \mu \mathrm{m} ;(-E, 200 \mu \mathrm{m}$.
Calbindin and $\mathrm{cSpV}$ terminations

Double labeling for sVTT fibers and calbindin (Fig. 6) revealed that fibers containing the anterogradely transported fluorescent tracer (green) terminate in thalamic areas often close to the densest region of calbindin-rich neuropil (red) in the medial tip of VPM but rarely coextensive with the latter. The principal clusters of $\mathrm{cSpV}$ axon terminations are located in an area inside VPM (Figs. 4, 5) but lateral to the calbindin-rich medial tip of the nucleus (Fig. 6A,C). The clusters of sVTT terminations occupy thalamic regions in and adjacent to VPM that are characterized by the presence of calbindin-immunopositive cells of the VPM matrix but are lacking in the dense calbindin-immunopositive fiber plexus of medial VPM (Fig. 6B). In double-labeled preparations, none of the axon terminations in the thalamus were found to be immunopositive for calbindin, confirming the result found previously (Rausell et al. 1992).

\section{$\mathrm{cSpV}$ thalamic fibers are not calbindin positive}

The fibers arising from the injection sites in the $\mathrm{cSpV}$ were followed throughout their course to the thalamus (Figs. $2 B, 7$ ). In the medullary trigeminothalamic tract ipsilateral to the injection site (Fig. 7A), a dense bundle of labeled fibers (green), which were calbindin negative, ran between dense bundles of calbindin-positive fibers, which were completely devoid of fluorescent tracer. Labeled fibers crossing to the contralateral side traversed the midline in the dorsal or ventral lemniscal decussation (data not shown). More rostrally in the brainstem, at the level of the pontine nuclei (Fig. $7 B$ ), the labeled fibers ran along the dorsolateral aspect of the medial lemniscus and ventral to the pontine reticular formation and accessory superior olivary nucleus, which are rich in calbindinimmunoreactive fibers and cells. Further rostrally in the brainstem, sVTT labeled fibers lay ventral to the lateral lemniscus, in proximity to calbindin-positive fibers of the lateral tegmental pathways (Fig. 7C). Along the length of the pathway from the $\mathrm{cSpV}$ to the thalamus, none of the fluorescent-labeled sVTT fibers were calbindin immunopositive (Figs. 6, 7).

\section{Antibody specificity}

We used Western blot analysis to compare the activity of the anti-D-28k calbindin antiserum used in the present and previous studies (Hendry et al., 1989; Jones and Hendry, 1989; Rausell and Jones, 1991a,b; Rausell et al., 1992; Jones et al., 2001) with 
a commercial monoclonal antibody used by Craig and colleagues (Craig et al., 1994, 2002; Blomqvist et al., 2000; Craig and Blomqvist, 2002). On immunoblots of total protein lysate from adult rhesus monkey thalamus, both antibodies recognized a single band of $\sim 28 \mathrm{kDa}$ (Fig. 8, right). We then immunostained sections from rhesus monkey brain with both primary antibodies, using two different secondary antibodies (Fig. 8, left). The confocal microscopy images showed an almost complete superimposition of all immunostained structures (Fig. 8C, yellow). Both antibodies revealed calbindin immunoreactivity in cells as well as in fibers and neuropil. Although the immunostaining obtained with the two antibodies overlapped almost completely, the polyclonal antiserum (Fig. 8, red) immunostained a slightly more extensive area of neuropil than the monoclonal antibody (Fig. 8, green).

\section{Discussion}

The main findings of this study are: (1) immunocytochemistry confirmed that the localized focus of densest calbindin immunoreactivity in the posterior thalamus, identified by others as VMpo, is part of a larger zone of dense calbindin immunoreactivity that lies well within the borders of the VPM, as delineated by other stains; (2) sVTT terminations are widespread and not restricted to the calbindin-rich focus in the medial tip of the VPM; (3) anterogradely labeled sVTT fibers and their terminations do not express detectable amounts of calbindin; and (4) two anti-calbindin antibodies, previously argued to be the source of differing interpretations of sVTT terminations in the thalamus, recognize identical epitopes. From these findings, we conclude that VMpo has no existence as a specific thalamic nucleus, and that there is no dedicated calbindin-labeled pathway running from superficial spinal and medullary dorsal horn that relays pain and temperature messages via a restricted calbindin-defined zone to insular and cingulate cortex.

\section{Calbindin in the posterior thalamus: the neurochemistry issue}

Certain facts about calbindin immunostaining in the thalamus have to be ignored to accept VMpo as an entity. Calbindin staining in and around the posterior pole of the VP nuclei is more extensive than that found in the densest focus at the medial tip of VPM that is identifiable with VMpo (Rausell and Jones, 1991a,b; Rausell et al., 1992; Jones, 1997, 1998; Morel et al., 1997; Jones et al., 2001), although the tip might appear as a localized focus in weakly immunostained preparations. The zone envisioned as VMpo by Craig et al. (1994), when adequately stained, is embedded in a larger area of dense calbindin immunoreactivity that is an integral part of VPM.

Previous studies of calcium-binding protein immunoreactivity in monkey thalamus located the densest focus of calbindinimmunoreactive cells and fibers within VPM and were coextensive with the medial rods of dense parvalbumin immunoreactivity and CO staining, and this was confirmed in the present study. The rods
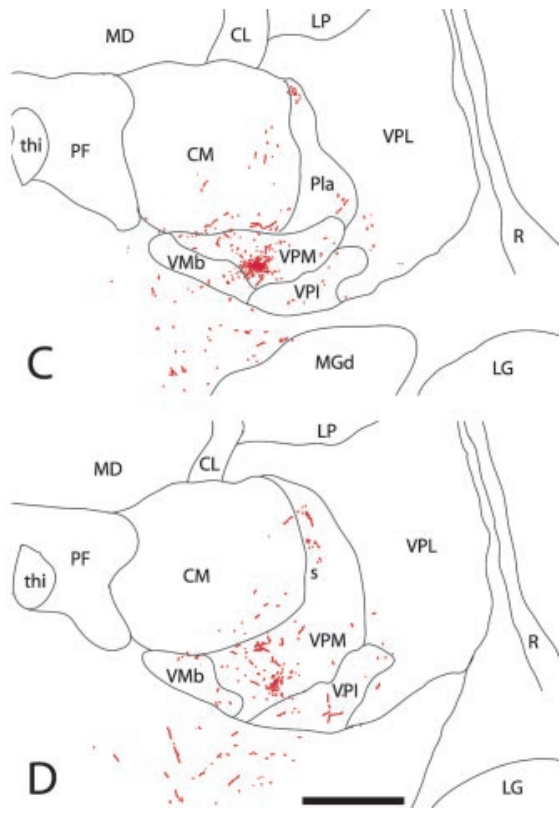

LG

Figure 4. $A, D$, Drawings from MD2 plottings of serial frontal sections of the posterior thalamus contralateral to the injection site, 作 of nuclei in the posterior thalamus. Note that the densest duster of terminations in C, although forming a well defined zone, is well inside posterior nucleus; MD, mediodorsal nucleus; MGd, dorsal medial geniculate nucleus; PF, parafascicular nucleus; $\mathrm{Pi}$, inferior pulvinar nu-celled zone of VPM nucleus; thi, habenulopeduncular tract. Scale bar, $1 \mathrm{~mm}$.
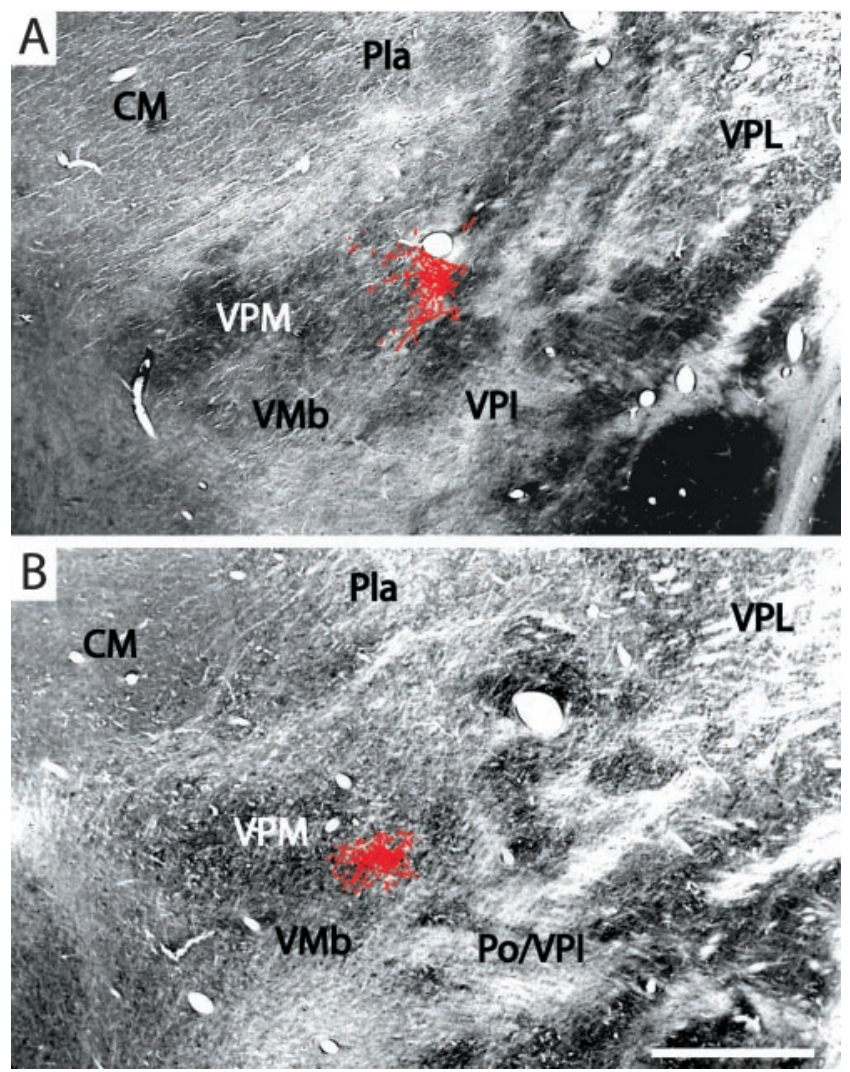

Figure 5. Frontal sections through the medial tip of VPM stained for $\mathrm{C} O$ with superimposed plottings of the principal clusters of labeled sVTT fiber terminations. The patches of dense $\mathrm{CO}$ staining correspond to the rods of VPM as seen in Figure 1, and the labeled terminations lie inside the boundaries of VPM, lateral to its medial tip, in which the dense $\mathrm{CO}$ staining corresponds to the region of densest calbindin immunostaining (Fig. 1). Scale bar, $1 \mathrm{~mm}$. 

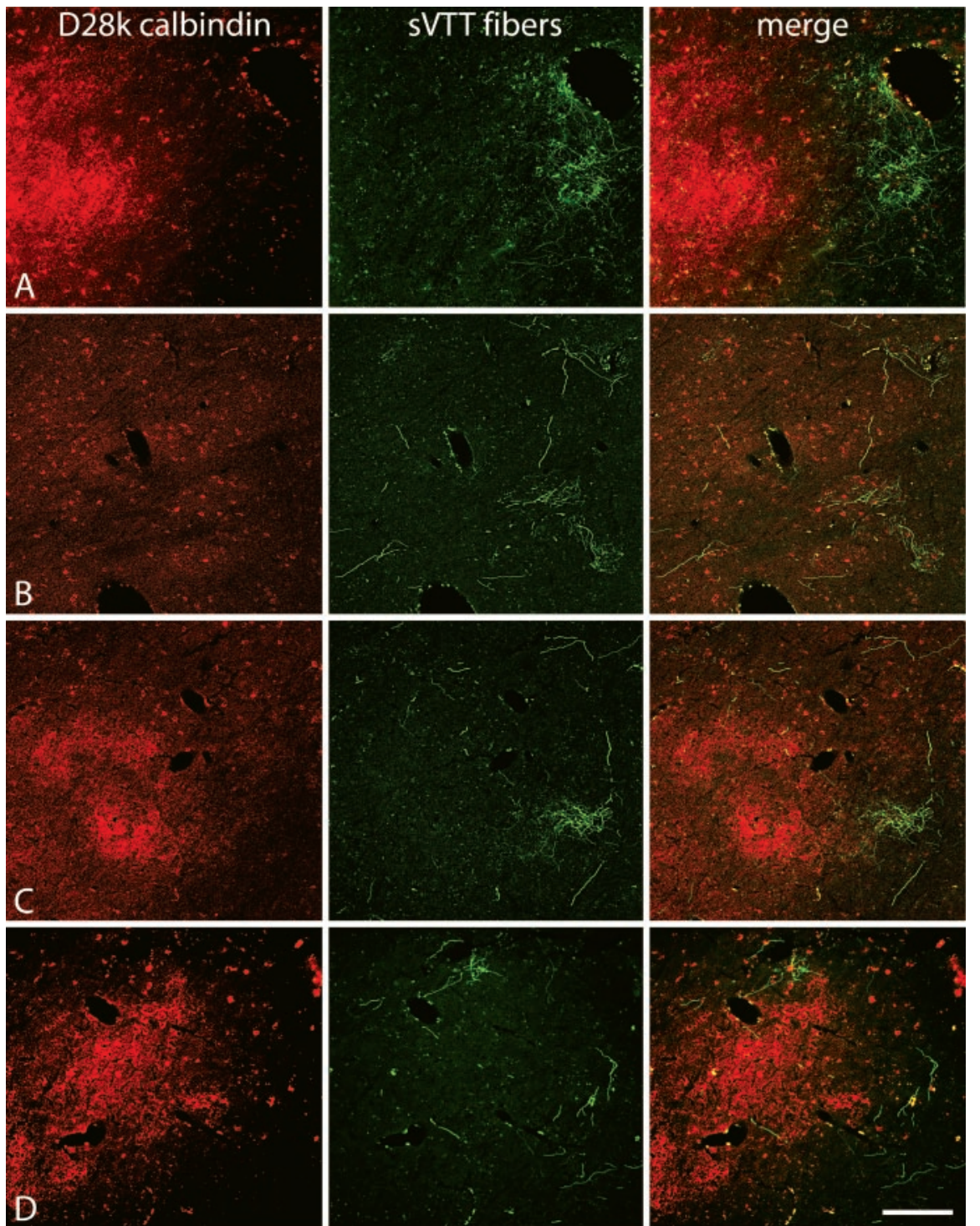

Figure 6. Dual-channel laser confocal micrographs from frontal sections showing the distribution of labeled sVTT terminations (green) in relation to the region of densest calbindin immunostaining (red) in the medial tip of VP. The labeled fibers terminate close to the region of calbindin-rich neuropil but rarely coextensive with it. $A, C, D$, The principal clusters of terminations lie lateral to the calbindin-rich medial tip of VPM. B, Some clusters of terminations are located in VPM areas characterized by calbindinimmunopositive cells of the matrix but lacking the dense calbindin-immunopositive fiber plexus at the medial tip of VPM. Scale bar, $400 \mu \mathrm{m}$.

are the targets of trigeminal lemniscal fibers, and the most medial ones contain neurons with intraoral receptive fields (Rausell and Jones, 1991a,b). Later studies in monkeys and humans confirmed the extent of calbindin immunostaining in VPM (Morel et al., 1997; Münkle et al., 2000; Jones et al., 2001). Although the VMb and VPI nuclei within the VP complex and the Pla and Po outside it show many calbindin-immunoreactive cells and fibers, the densest concentration of calbindin-immunopositive fibers remains well within the medial tip of VPM. The delineation of VMpo as a distinct entity outside the VP complex seems to have depended on observations made without recourse to the evidence of staining patterns derived from the use of other markers. The dense calbindin fiber plexus in the medial tip of VPM stands out in weakly immunostained single sections, but more comprehensive immunostaining and complementary staining of the same or adjacent sections over the full rostrocaudal extent of this region of the thalamus show its location well inside the boundaries of VPM. There is no denser focus of calbindin immunoreactivity outside the medial tip of VPM, and thus VMpo cannot be argued as part of any other nuclear complex such as the posterior group.

Differences between descriptions of calbindin in the posterior thalamus reside in the overall quality and intensity of immunostaining and in the limited sampling of immunostained sections by Craig et al. (1994) and Blomqvist et al. (2000). They cannot be attributed to the use of different anti-calbindin antibodies, because we show that both antibodies recognize a single band of appropriate molecular weight $(\sim 28 \mathrm{kDa})$, and immunostaining by the two on the same sections was completely superimposed. The only difference was a slightly broader staining of the neuropil with the polyclonal antiserum, probably because of higher antibody titer and possibly because this also recognizes $29 \mathrm{kDa}$ calretinin, the expression of which overlaps that of calbindin in the posterior thalamus (Jones et al., 2001; http://neuroscience.ucdavis.edu/ Jones/ThalamusImages/Monkey/). Because the antibody that we used immunostained an area slightly more extensive than that revealed by the commercial antibody, the probability of finding superficial sVTT terminations in calbindin-immunopositive areas should have been enhanced. Nevertheless, we did not find any in the dense medial tip of VPM. The anterograde tracers used also cannot account for the different results of the tracing studies, for those used in the separate studies have similar sensitivities (Dolleman-Van der Weel et al., 1994; Vercelli et al., 2000).

\section{Lamina I projections to the thalamus: the connectivity issue}

In the brief description on which their idea of a pain- and temperature-specific thalamic nucleus innervated by lamina I neurons from the full length of the dorsal horn is based, Craig et al. (1994) mentioned the labeling of spinothalamic axons by injection of tracers restricted to lamina I but provided no documentation. Given the thinness of lamina I, it seems unlikely that injections sufficiently large to label a significant number of projecting axons could be placed without involvement of at least lamina II as well. Our approach was to make injections that would involve relatively large parts of lamina I in conjunction with lamina II, arguing that if lamina I projected to the calbindin-rich medial tip of the VP complex, these projections would be evident in the overall pattern of labeling obtained. It was striking that none of the terminal ramifications labeled in our experiments occupied the most calbindinrich part of the VP complex that represents what Craig et al. (1994) called VMpo. Instead, the principal cluster of terminations was located lateral to the calbindin-rich zone, and other clusters were scattered throughout other parts of VP and neighboring nuclei. Although these results do not rule out the possibility that injections of tracer involving lamina I in other parts of 
the spinal cord and $\mathrm{cSpV}$ could label axons projecting to the calbindin-rich medial tip, they rule out the belief put forward by Craig et al. (1994) that lamina I cells in all parts of the spinal and medullary dorsal horns, and representing all parts of the body, project their axons only to the richest calbindin area in posteromedial VP. It seems likely that the injections described by Craig et al. (1994) were too small and of too limited a rostrocaudal extent to reveal the complex and widespread pattern of projections from lamina I to the thalamus (Jones, 2002; Willis et al., 2002; Ralston, 2003); however, the account of labeled terminations of STT and sVTT axons representing the face, body, and limbs being found exclusively in the calbindin-rich tip of VPM cannot be correct. From mapping studies, the medial tip represents only ipsilateral intraoral structures (Rausell and Jones, 1991a) and projects to primary somatosensory cortex (Manger et al., 1995, 1996), not to the cingulate region.

Retrograde tracing studies reveal that many cells in lamina I of the spinal cord and $\mathrm{cSpV}$ can be labeled from a broad area of the contralateral thalamus, including the principal sensory relay nuclei, VPM, and VPL (Willis and Westlund, 1997; Willis et al., 2002). Injections in the central core of VP, sparing the area containing the putative VMpo, resulted in a significant number of retrogradely labeled cells in lamina I of the $\mathrm{cSpV}$ and spinal cord dorsal horn (Willis et al., 2001). Data derived from single-unit recordings showed neurons with noxious and thermal-specific stimulus-response properties, some of which projected to the primary somatosensory cortex scattered throughout VPM and VPL (Applebaum et al., 1979; Casey and Morrow, 1983; Honda et al., 1983;

Kenshalo and Isensee, 1983; Chung et al., 1986; Chandler et al., 1992; Apkarian and Shi, 1994; Ohara and Lenz, 2003). Retrograde labeling of cells in lamina I from injections involving "VMpo" was based on extensive injections that were not confined to the medial tip of the VP complex (Craig et al., 2002). Thus, the weight of evidence is against the idea of a spinothalamocortical pathway for pain and temperature sensation that disregards the principal thalamic sensory relay nuclei, VPL, and VPM, and, as a corollary, the primary somatosensory cortex.

\section{Lamina I projections to the thalamus: the calbindin issue}

Craig et al. $(1994,2002)$ and Blomqvist et al. (2000) proposed that the pain pathway is characterized by the presence of high levels of calbindin immunoreactivity but gave no data on the basis of double-labeling strategies to support this. Although appealing in its simplicity, this view is in marked contrast to the present and previous studies (Rausell et al., 1992) in which it was not possible to double label any anterogradely labeled STT fibers or their thalamic terminations for calbindin. There is still no formal evidence that STT fibers express calbindin, or that calbindin cells of lamina
I project to the thalamus. Although the presence of a concentrated zone of calbindin-positive fibers in the dorsal part of the spinal lateral funiculus and in the medial tip of the VP complex invite the speculation that the one contains fibers that terminate in the other, this is now ruled out. It has not been ascertained where the calbindin-positive fibers of the lateral funiculus arise and project. In the rat spinal cord, Li et al. (1999) found no obvious correlation between the targets of lamina I cells (thalamic, hypothalamic, or parabrachial regions) and the calciumbinding proteins expressed.

In the thalamus, the high density of calbindin fibers in the medial tip of the VP complex is associated with a high density of calbindin-immunoreactive cells, all of which have cortically projecting axons (Rausell et al., 1992). The dense calbindin fiber plexus that is postulated to be the hallmark of VMpo is as much made up of these immunolabeled thalamocortical axons, as it is of the terminal ramifications of calbindin-immunoreactive fibers entering from the midbrain tegmentum (Jones et al., 2001). The origins of these tegmental fibers remain to be determined; how- 

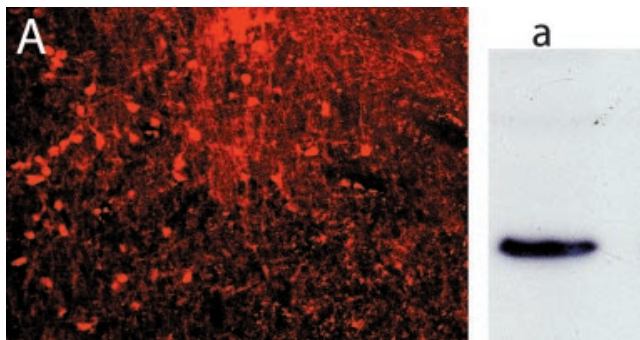

b
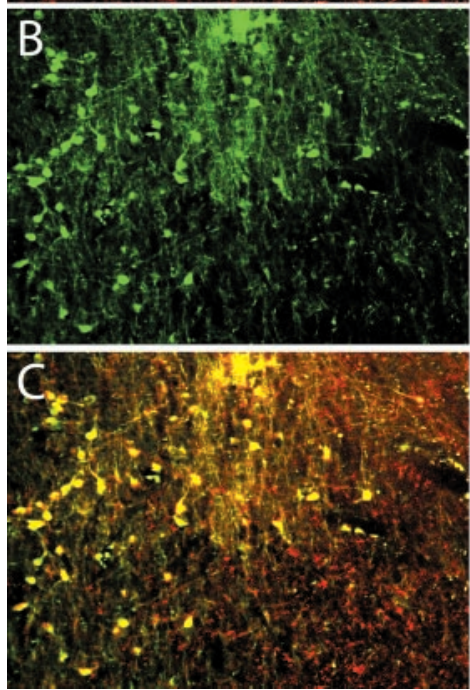

Figure 8. Antibody specificity. Right, Western blot of total protein lysate from adult rhesus monkey thalamus. $A, B$, Both the commercial monoclonal $(A)$ and polyclonal $(B)$ anti-calbindin antibodies recognized a single band of $\sim 28 \mathrm{kDa}$. Left, Dual-channel laser confocal micrographs of a rhesus monkey brain section immunostained with both anti-calbindin antibodies; the immunostaining obtained with the polyclonal $(A)$ and the commercial monoclonal antibody $(B)$ are merged in $C$, where almost complete overlap is evident. Note that the polyclonal antiserum immunostained a slightly broader area of neuropil ( $C$, red) than the monoclonal antibody.

ever, as shown by the present results, they do not arise exclusively or at all from the medullary dorsal horn.

\section{References}

Apkarian AV, Hodge CJ (1989a) Primate spinothalamic pathways. I. A quantitative study of the cells of origin of the spinothalamic pathway. J Comp Neurol 288:447-473.

Apkarian AV, Hodge CJ (1989b) Primate spinothalamic pathways. II. The cells of origin of the dorsolateral and ventral spinothalamic pathways. J Comp Neurol 288:474-492.

Apkarian AV, Hodge CJ (1989c) Primate spinothalamic pathways. III. Thalamic terminations of the dorsolateral and ventral spinothalamic pathways. J Comp Neurol 288:493-511.

Apkarian AV, Shi T (1994) Squirrel-monkey lateral thalamus. I. Somatic nociresponsive neurons and their relation to spinothalamic terminals. J Neurosci 14:6779-6795.

Applebaum AE, Leonard RB, Kenshalo DR, Martin RF, Willis WD (1979) Nuclei in which functionally identified spinothalamic tract neurons terminate. J Comp Neurol 188:575-585.

Blomqvist A, Zhang ET, Craig AD (2000) Cytoarchitectonic and immunohistochemical characterization of a specific pain and temperature relay, the posterior portion of the ventral medial nucleus, in the human thalamus. Brain 123:601-619.

Bradford MM (1976) Rapid and sensitive method for quantitation of microgram quantities of protein utilizing principle of protein-dye binding. Anal Biochem 72:248-254.

Casey KL, Morrow TJ (1983) Ventral posterior thalamic neurons differentially responsive to noxious-stimulation of the awake monkey. Science 221:675-677.

Chandler MJ, Hobbs SF, Fu QG, Kenshalo DR, Blair RW, Foreman RD
(1992) Responses of neurons in ventroposterolateral nucleus of primate thalamus to urinary-bladder distension. Brain Res 571:26-34.

Chung JM, Lee KH, Surmeier DJ, Sorkin LS, Kim J, Willis WD (1986) Response characteristics of neurons in the ventral posterior lateral nucleus of the monkey thalamus. J Neurophysiol 56:370-390.

Craig AD, Blomqvist A (2002) Is there a specific lamina I spinothalamocortical pathway for pain and temperature sensations in primates? J Pain 3:95-101.

Craig AD, Burton H (1981) Spinal and medullary lamina-I projection to nucleus submedius in medial thalamus-a possible pain center. J Neurophysiol 45:443-466.

Craig AD, Bushnell MC, Zhang ET, Blomqvist A (1994) A thalamic nucleus specific for pain and temperature sensation. Nature 372:770-773.

Craig AD, Zhang ET, Blomqvist A (2002) Association of spinothalamic lamina I neurons and their ascending axons with calbindin-immunoreactivity in monkey and human. Pain 97:105-115.

Dolleman-Van der Weel MJ, Wouterlood FG, Witter MP (1994) Multiple anterograde tracing, combining Phaseolus-vulgaris leukoagglutinin with rhodamine-conjugated and biotin-conjugated dextran-amine. J Neurosci Methods 51:9-21.

Getz B (1952) The termination of spinothalamic fibers in the cat studied by the method of terminal degeneration. Acta Anat (Basel) 16:271-290.

Hendry SHC, Jones EG, Emson PC, Lawson DEM, Heizmann CW, Streit P (1989) Two classes of cortical gaba neurons defined by differential calcium-binding protein immunoreactivities. Exp Brain Res 76:467-472.

Hodge CJ, Apkarian AV (1990) The spinothalamic tract. Crit Rev Neurobiol 5:363-397.

Honda CN, Mense S, Perl ER (1983) Neurons in ventrobasal region of cat thalamus selectively responsive to noxious mechanical stimulation. J Neurophysiol 49:662-673.

Jones EG (1985) The thalamus. New York: Plenum.

Jones EG (1997) A description of the human thalamus. In: Thalamus (Steriade $\mathrm{M}$, Jones EG, McCormick DA, eds), pp 425-499. Amsterdam: Elsevier.

Jones EG (1998) The primate nervous system, Part II. In: Handbook of chemical neuroanatomy, Vol. 14 (Björklund A, Bloom FE, Hökfelt T, eds), pp 1-298. Amsterdam: Elsevier.

Jones EG (2002) A pain in the thalamus. J Pain 3:102-104

Jones EG, Hendry SHC (1989) Differential calcium-binding protein immunoreactivity distinguishes classes of relay neurons in monkey thalamic nuclei. Eur J Neurosci 1:222-246.

Jones EG, Lensky KM, Chan VH (2001) Delineation of thalamic nuclei immunoreactive for calcium-binding proteins in and around the posterior pole of the ventral posterior complex. Thalamus and Related Systems 1:213-224.

Kenshalo DR, Isensee O (1983) Responses of primate SI cortical-neurons to noxious stimuli. J Neurophysiol 50:1479-1496.

Kniffki KD, Mizumura K (1983) Responses of neurons in VPL and VPL-VL region of the cat to algesic stimulation of muscle and tendon. J Neurophysiol 49:649-661.

Laemmli UK (1970) Cleavage of structural proteins during the assembly of the head of bacteriophage T4. Nature 227:680-685.

Li JL, Li YQ, Li JS, Kaneko T, Mizuno N (1999) Calcium-binding proteinimmunoreactive projection neurons in the caudal subnucleus of the spinal trigeminal nucleus of the rat. Neurosci Res 35:225-240.

Manger PR, Woods TM, Jones EG (1995) Representation of the face and intraoral structures in area 3B of the squirrel-monkey (Saimiri sciureus) somatosensory cortex, with special reference to the ipsilateral representation. J Comp Neurol 362:597-607.

Manger PR, Woods TM, Jones EG (1996) Representation of face and intraoral structures in area $3 \mathrm{~b}$ of macaque monkey somatosensory cortex. J Comp Neurol 371:513-521.

Mehler WR, Feferman ME, Nauta WJH (1960) Ascending axon degeneration following anterolateral cordotomy: an experimental study in the monkey. Brain 83:718-751.

Morel A, Magnin M, Jeanmonod D (1997) Multiarchitectonic and stereotactic atlas of the human thalamus. J Comp Neurol 387:588-630.

Münkle MC, Waldvogel HJ, Faull RLM (2000) The distribution of calbindin, calretinin and parvalbumin immunoreactivity in the human thalamus. J Chem Neuroanat 19:155-173. 
Ohara S, Lenz FA (2003) Medial lateral extent of thermal and pain sensations evoked by microstimulation in somatic sensory nuclei of human thalamus. J Neurophysiol 90:2367-2377.

Olszewski J (1952) The thalamus of the Macaca mulatta: an atlas for use with stereotaxic instruments. Basel: Karger.

Price DD, Dubner R, Hu JW (1976) Trigeminothalamic neurons in nucleus caudalis responsive to tactile, thermal, and nociceptive stimulation of monkeys face. J Neurophysiol 39:936-953.

Ralston HJ (2003) Pain, the brain, and the (calbindin) stain. J Comp Neurol 459:329-333.

Ralston HJ, Ralston DD (1994) Medial lemniscal and spinal projections to the macaque thalamus: an electron-microscopic study of differing GABAergic circuitry serving thalamic somatosensory mechanisms. J Neurosci 14:2485-2502.

Rausell E, Jones EG (1991a) Chemically distinct compartments of the thalamic VPM nucleus in monkeys relay principal and spinal trigeminal pathways to different layers of the somatosensory cortex. J Neurosci 11:226-237.
Rausell E, Jones EG (1991b) Histochemical and immunocytochemical compartments of the thalamic VPM nucleus in monkeys and their relationship to the representational map. J Neurosci 11:210-225.

Rausell E, Bae CS, Viñuela A, Huntley GW, Jones EG (1992) Calbindin and parvalbumin cells in monkey VPL thalamic nucleus: distribution, laminar cortical projections, and relations to spinothalamic terminations. J Neurosci 12:4088-4111.

Vercelli A, Repici M, Garbossa D, Grimaldi A (2000) Recent techniques for tracing pathways in the central nervous system of developing and adult mammals. Brain Res Bull 51:11-28.

Willis WD, Westlund KN (1997) Neuroanatomy of the pain system and of the pathways that modulate pain. J Clin Neurophysiol 14:2-31.

Willis WD, Zhang X, Honda CN, Giesler GJ (2001) Projections from the marginal zone and deep dorsal horn to the ventrobasal nuclei of the primate thalamus. Pain 92:267-276.

Willis WD, Zhang XJ, Honda CN, Giesler GJ (2002) A critical review of the role of the proposed VMpo nucleus in pain. J Pain 3:79-94. 